1895. Mille huit cent quatre-vingt-quinze

Revue de l'association française de recherche sur

I'histoire du cinéma

$61 \mid 2010$

Aux sources du burlesque cinématographique : les comiques français des premiers temps

\title{
Scènes primitives. Notes sur quelques genres comiques « hérités » du café-concert
}

Thomas Louis Jacques Schmitt

\section{(2) OpenEdition \\ Journals}

Édition électronique

URL : https://journals.openedition.org/1895/3838

DOI : $10.4000 / 1895.3838$

ISBN : 978-2-8218-0980-2

ISSN : $1960-6176$

Éditeur

Association française de recherche sur l'histoire du cinéma (AFRHC)

Édition imprimée

Date de publication : 1 septembre 2010

Pagination : 174-190

ISBN : 978-2-913758-62-9

ISSN : 0769-0959

\section{Référence électronique}

Thomas Louis Jacques Schmitt, «Scènes primitives. Notes sur quelques genres comiques « hérités » du café-concert », 1895. Mille huit cent quatre-vingt-quinze [En ligne], 61 | 2010, mis en ligne le 01 septembre 2013, consulté le 16 avril 2022. URL : http://journals.openedition.org/1895/3838; DOI : https://doi.org/10.4000/1895.3838 


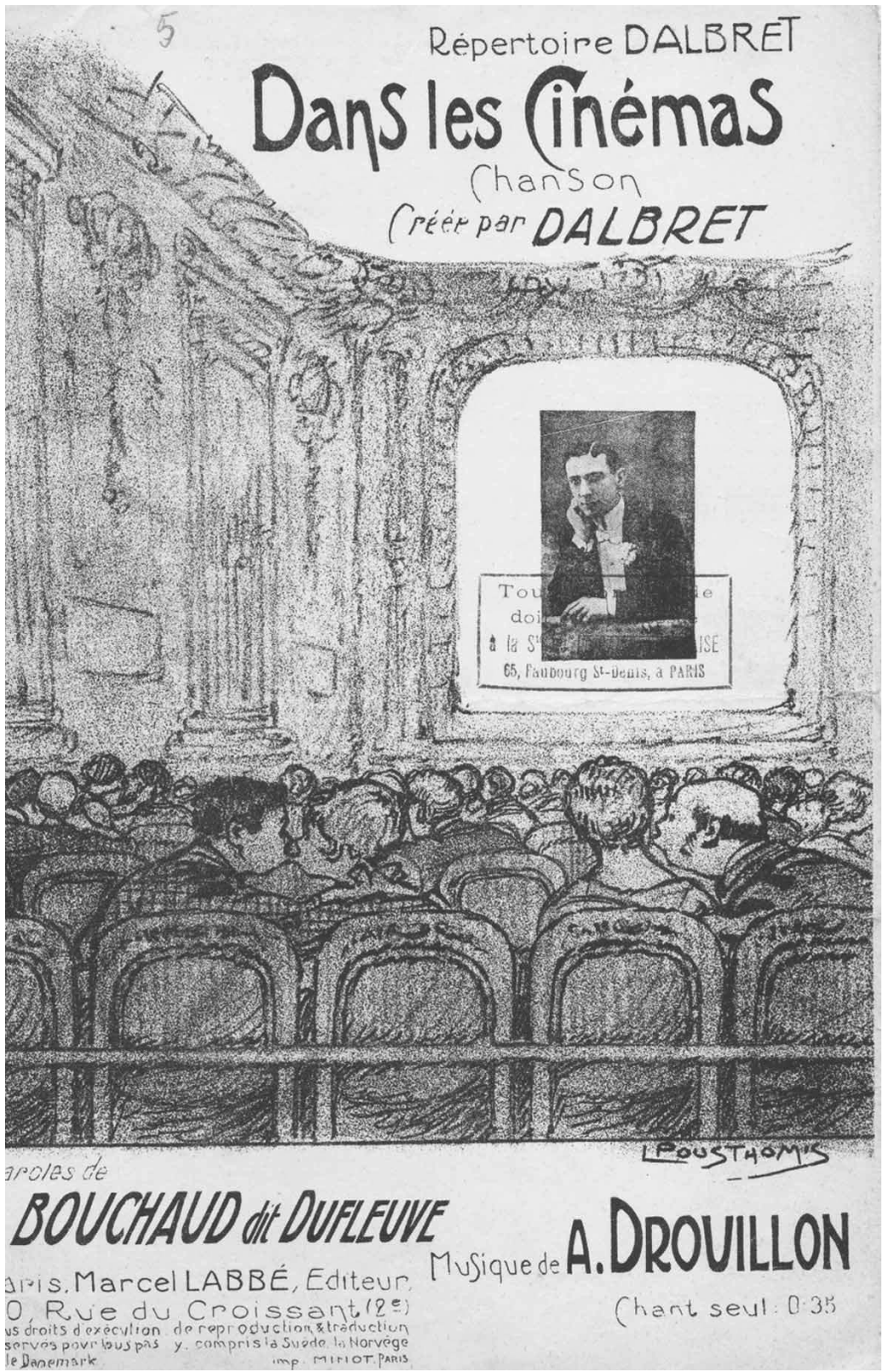

Fig. 1. Dans les cinémas. Paroles de Bouchaud (dit Dufleuve), musique de A. Drouillon. Petit format sans date. Répertoire Dalbret. Illustration de L. Pousthom's. Marcel Labbé Éditeur, 20 rue du Croissant, Paris. Dédié à Gilbert Darsay, directeur du Palais de Cristal. 


\section{Scènes primitives. \\ Notes sur quelques genres comiques \\ " hérités " du café-concert}

Thomas Louis Jacques Schmitt

Les effets de répétition présentent quelquefois cette forme spéciale au théâtre et dans le roman : certains d'entre eux ont des résonances de rêve. Et peut-être en est-il de même du refrain de bien des chansons : il s'obstine, il revient, toujours le même, à la fin de tous les couplets, chaque fois avec un sens différent.

Henri Bergson, le Rire.

Cet article ne prétend pas révéler une quelconque « origine » des genres comiques du cinéma des premiers temps. Les « scènes primitives » que nous évoquerons par la suite ne nous semblent pas avoir été directement transposées de la rampe à l'écran. Au contraire, la principale thèse avancée ici propose que l'arrivée du cinématographe ait très tôt interagi avec la chanson de café-concert ou la revue de music-hall, comme ce sera plus tard le cas avec la TSF. Qu'ils aient été projetés sur l'écran sous la forme de " phonoscènes " ou entonnés sur la scène, les genres de la chanson comique répondent d'après nous à un modèle de contamination réciproque. Le premier couplet de Cinémato parisien enregistré en 1903 par Charlus ${ }^{1}$ illustre cette hypothèse :
Un spectacle qui jamais ne lasse
C'est d'voir sur les boulevards
Les badauds, les jobards
Les attroupements, les gens qui passent
C'est un cinémato très rigolo

1 Paroles : Léo Lelièvre et Henri Christiné. Musique : Neil Moret "Hiawatha". Disque Odéon ITM 36297. Transcription de l'auteur. 
Ce « cinémato » semble un fait culturel suffisamment intégré vers 1903 dans le paysage social pour qu'il puisse faire l'objet d'une chanson. Certes, Charlus ne chante pas encore le sixième Art dont Canudo annonce la naissance en 19112, le ciné de Colette ${ }^{3}$ ou le cinéma \& Cie de Delluc, mais il ne fredonne déjà plus exactement le cinématographe fin de siècle. Ainsi, dans le couplet suivant de la chanson, Charlus décrit à distance des genres, des types, des caractères, comme le ferait le bonimenteur comique d'une vue Lumière. Sur l'écran imaginaire tressaute un drôle de réel, synchrone des stéréotypes des premières bandes comiques Pathé ou Gaumont :

Le vieux beau toujours fringant

Frétillant, épaulant,

Suit la petite ouvrière

Tralala lalala...

Et le trottin gentiment,

Se r'tournant, dit en riant

"Va donc remiser ta cafetière "

Tralala lalala...

L'écran rendrait-il la réalité comique comme le phonographe ridiculise la voix humaine ? L'irruption de l'enregistrement mécanique redéfinit la frontière entre réalité et représentation, scène et public. Le « cinémato » est une machinerie qui - passés les tout premiers temps d'émerveillements scientifico-forains - plaque automatiquement du ridicule sur le vivant, mécanisant la gestuelle filmée. De plus, en rendant visibles des « images » jusque-là uniquement énoncées en paroles, le « cinémato » questionne les limites de la bienséance et les " manières de voir ». Le " cinémato » change non seulement l'offre de divertissement mais aussi les pratiques spectatorielles. Dans les cinémas, chanson de Dufleuve (fig. 1), décrit ainsi la rivalité qui oppose les spectacles chantés et projetés. La chanson rend compte dans le même temps des nouvelles pratiques de promiscuité que permettent les « salles obscures " par rapport aux cafés-concerts :

2 Roman Gubern, "L'histoire du cinéma par rapport à ses théories esthétiques », dans Irène Bessière et Jean A. Gili (dir.), Histoire du cinéma. Problématique des sources, Paris, AFRHC, 2004, p. 271.

3 Colette, "Cinéma : Forfaiture » dans Excelsior, 7 août 1916. 
Si vous voulez vous amuser

Allez au cinématographe

Dans tous ces jolis Kinograph's

Et dans ces beaux cinématographes

Vous n'entendez pas des chansons,

comm' cell's qu'on chant' dans les concerts

On y donne un spectacle sain,

où I'on n'entend rien de pervers

Vous verrez sur la toile

Une petit' femm' sans voile

Dans les bras de son cher amant

Lui donnant des baisers brûlants

Puis vous verrez l'époux

Le cornard en courroux

Qui surprend sa femme au dodo

En train de chanter un duo

Comm' vous voyez, c'est très moral

Et quand j'veux m'offrir un régal

Je vais dans les cinémas,

Kinémas, Kinéramas

Lorsque la lumière est éteinte

De l'amour, je vois les étreintes

Je vais dans les cinémas,

Kinémas, kinéramas

C'est là que les plus farouches vertus

S'en vont voir les amours tous nus

[...]

Dans ces endroits bien sombres

On aperçoit dans l'ombre

Des mains qui s'faufil'nt doucement

Et disparaiss'nt sous les vêt'ments 
On entend des soupirs

Qu'on cherche à retenir

Dès qu'la salle s'éclaire c'est curieux

On ne voit que des gens sérieux

\section{$[\ldots]$}

Je vais dans les cinémas,

Kinémas, kinéramas

Je vois dans ces salles intimes

Le sang, le viol, la guillotine

[...]

Depuis que j'connais ces endroits charmants

Je trouve le concert dégoûtant

Ces nouveaux usages impliqués par le cinéma peuvent-ils être comptés parmi les causes de l'escalade du café-concert vers l'obscénité ? Observons rapidement la chronologie du phénomène dans le cas de Toulouse.

Le Palace Cinéma Théâtre, inauguré le 9 décembre 1906 au 41, rue Alsace-Lorraine est-il véritablement le " plus important cinématographe de province » comme l'annonce la Dépêche du Midi du 5 novembre $1907^{4}$ ? La direction de l'établissement tient en tout cas à préciser aux Toulousains, dans les nombreux communiqués qu'elle fait publier, que « le Palace Cinéma Théâtre ne donne que des spectacles de famille, toujours moraux, instructifs et amusants. ${ }^{5}$ Première salle permanente de la ville rose, le Palace Cinéma Théâtre, fondé par Napoléon Rancy, repris, agrandi et ré-inauguré le 20 octobre 1907 par un certain Mielliery, propose dans une même séance la Fontaine de jouvence, grande féerie en 25 tableaux, "dernière création de Gaumont " et Mortelle idylle, le « drame émouvant de Pathé ". De même, face au Théâtre du Capitol, au Théâtre Lafayette, au Théâtre des Nouveautés ${ }^{6}$, à une pléthore de cafés-concerts et face à des concurrents plus directs (American Cosmograph et Cinéma Pathé Royal Saint Cyprien), la communication du "Cinéma Alsace " (surnom donné au Palace Cinéma Théâtre par les Toulousains) tient à affirmer la spécificité de son programme : « Au total, 3000 m de vues défilent devant le spectateur émerveillé ; c'est le théâtre, l'école, le

4 Xavier Vadillo-Aurtenetxe, "Histoire du cinéma à Toulouse des origines à 1914 ", Munich, suppl. au $\mathrm{n}^{\circ} 21$ de la revue Cicim, Institut français de Munich, janvier 1988, p. 33.

5 La Dépêche du Midi, 4 janvier 1908.

6 Le Théâtre des Variétés a brûlé en mai 1907. 
journal pour tous. Souvent comique, parfois tragique, toujours moral. " "Or, si en janvier 1908, la Dépêche du Midi présente encore sous la même rubrique ("Spectacle à Toulouse ") tous les « théâtres " cinématographiques ou non, une distinction apparaît, en novembre de la même année, les trois salles de cinéma déjà citées abandonnant l'appellation de théâtre dans leurs communiqués. Mais c'est aussi le cas pour " Les Nouveautés » - un « vaisseau de 2500 places $»^{8}$ présentant des " numéros de cirque au goût pas toujours très raffiné ${ }^{9}$. Soucieux de se démarquer des cafés-concerts qui se lancent depuis 1898 dans " une surenchère vers l'obscène, glissant plutôt du côté de la vulgarité que de la grivoiserie »10, l'établissement se fixe un code de bonne conduite et présente, au mois de décembre 1907, une « intéressante et ingénieuse application du phonographe et du cinématographe combiné "11 : le Chronomégaphone Gaumont, qui diffuse des phonoscènes (fig. 2 et 3). Quelque mois plus tard, la municipalité menace de fermer les cafés-concerts pour cause d'obscénité. L'irruption du " spectacle muet " aurait-elle rendu plus chastes les oreilles des censeurs ?

Dès lors que l'on cesse de vouloir faire le point sur le seul plan cinématographique, la mécanisation du comique, et plus largement l'industrialisation du spectacle (et sa moralisation), semblent un phénomène contextuel de l'apparition du cinématographe. Au contact de l'archive, l'assomption « le 7e Art est l'un des principaux responsables de la quasi-disparition des établissements consacrés aux arts vivants "12 devient floue. C'est que « les arts vivants » sont objectivement une construction dialectique de l'apparition des « arts de l'enregistrement ». Les années 1907/1908 marqueraient-elles le point d'orgue d'un processus entamé avec l'inauguration de I'Olympia en 1893, officiellement premier music-hall français' ${ }^{13}$ ? Comme le résume la littérature commerciale de Gaumont, l'ère de la reproduction mécanique annonce de nouveaux profits :

Quelques esprits chagrins ou timorés ont été jusqu'à prétendre que notre Chronophone était la mort des artistes. Nous les remercions de cette marque de succès. Elle vaudrait à elle seule mieux que toutes les publicités; mais cependant nous voulons rassurer ces mêmes artistes. Est-ce que le phonographe a tué les chanteurs ? N'a-t-on pas prédit la même ineptie à son apparition ? Quel

7 La Dépêche du Midi, 11 janvier 1908. On reconnaît ici la célèbre phrase de Charles-François Dussaud, ingénieur-conseil chez Pathé, promue au rang de slogan programmatique par la société de Vincennes, notamment dans son catalogue d'août 1905. (NdE)

8 André Sallée et Philippe Chaveau, Music-hall et café-concert, Paris, Bordas, 1985, p. 188.

9 Xavier Vadillo-Aurtenetxe, op. cit., p. 9.

10 Ibid., p.10.

11 La Dépêche du Midi, 4 décembre 1907.

12 André Sallée et Philippe Chaveau, op. cit., p. 34.

13 François Caradec et Alain Weill, Le café-concert, Paris, Atelier Hachette/Massin, 1980, p.183. 
$1895 /$

$n^{\circ} 61$

septembre

2010

180
58

\begin{tabular}{|c|c|c|c|c|c|c|c|c|c|c|}
\hline $\mathrm{N}^{*}$ & $\begin{array}{c}\text { CODE } \\
\text { Télégraphique }\end{array}$ & & TITRES & & & & GENRE & $\begin{array}{c}\text { LONG } \\
\text { approx. } \\
\text { en } \\
\text { metres }\end{array}$ & $\begin{array}{c}\text { PRIX } \\
\text { de la } \\
\text { bande en noir }\end{array}$ & $\begin{array}{l}\text { PRIX } \\
\text { d un } \\
\text { disque }\end{array}$ \\
\hline 138 & Sitire. & Polin. & Situalion intéressante & . & & & Wosologie fi fraxgals. & ¿9 & 147.50 & 5. \\
\hline 139 & Lelut. & $: \quad-$ & Chez les lutteurs & & & & 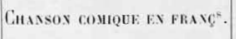 & 48 & 120 & $5 . "$ \\
\hline 140 & Cuisi. & - & La belle cuisinière. . . . & . . & . & & - & 52 & 130. & 5. " \\
\hline 141 & Rapor. & - & La leclure du rapport & . & . & & Wovotogee ex frangals. & 76 & $190 . n$ & i. " \\
\hline 142 & Scandi. & Scandi & linave..... & . & . & & Dasse. & 58 & 145. & ว. " \\
\hline 143 & Fifille. & Mayol & 1. La fifille à sa mère. . & . . & . & & CHarson comete a Faxes. & 48 & 120 & ว. . \\
\hline 144 & Plipe. & - & Le pelil panier & . & . & & - & if & 140 & 5. \\
\hline 145 & Tigre. & - & Le pelit Grégoire. . & . & . & & - & $\therefore 4$ & 140 & $5 . x$ \\
\hline 146 & Mapoul. & $*$ & Viens, Poupoule. . . . & . & . & & - & ;.5 & 137.50 & ذ. \\
\hline 147 & Lila. & $\therefore \quad-$ & Lilas blanc. . & . & . & & Chassos thiste es fruscais. & ti5 & 157.50 & 5. \\
\hline 148 & Lomai. & $\underline{-}$ & Jeune homme et trottin. & . . & . & & CIIS SOX COMOLE RX FRAX's. & 62 & 155. , & 5. " \\
\hline 149 & Maloy. & - & La polka des trollins. . & . . & . & & & 52 & 130 & 5. " \\
\hline 150 & Pimpol. & $\therefore \quad-$ & La Paimpolaise & . & . & & CHASSON BRETOXNE EA FRANC & 56 & 140. & 5. \\
\hline 151 & Ingen. & - & C'est une ingénue... . & . & . . & & CIMSOS COBIQUE FN RBAsç. & 56 & 140. & 5. \\
\hline 152 & Sateva, & - & Si ça t'va. . . & . & . & & - & 51 & 127.50 & 5. \\
\hline 153 & Bambou. & $\therefore-$ & A la cabane bambou. . . & . & . . & & - & 55 & 132.50 & 5. " \\
\hline 154 & Indis. & $\because$ & Questions indiscrètes. . & . & . & & - & 56 & 140 & 5. \\
\hline 155 & Matisti. & $* \quad-$ & La Mattchiche. . . . . & & . & & - & 50 & 125. & ¿. . \\
\hline 156 & Amlet. & Ballet & d'Hamlet. . . & . & . & & Daxse. & 60 & 150. & 5. " \\
\hline 157 & Alum. & Drane & em. Allumeur-Marche. & & . & & CHassos comequ ra flase. & 住 & 112.50 & 7.25 \\
\hline 158 & Trouq. & $* \quad-$ & Le trou de mon quai. & . . & . & & - & 19) & 122.50 & 7. 25 \\
\hline 159 & Lason. & -- & Valsons. & . & . & & 一 & 46 & 115. & 7.25 \\
\hline 160 & Retam. & $1=$ & V'la le rélameur. & & 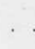 & & - & il & 127.50 & 5.75 \\
\hline 161 & Tipoi. & $\cdots$ & Les p'tits pois & & . & & & 41 & 102.50 & 5.75 \\
\hline 162 & Cordo. & - & L'enfant du cordonnie & $\mathrm{er}$. & . & & - & 45 & 112.50 & 5.75 \\
\hline 163 & Gume, & - & Être légume. . & 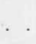 & . & & - & 58 & 95. " & 5.75 \\
\hline 164 & llace. & $* \quad-$ & Le cucurbilacée. . & & - & & - & 44 & 110 & .5. \\
\hline 165 & Cosmop. & 一 & Le boléro cosmopolite. & $\because$ & . & & - & 49 & 122.50 & 5. \\
\hline
\end{tabular}

Fig. 2 et 3. Catalogue de la Société des Établissements Gaumont. Sujets proposés à la vente en janvier 1908. Phonoscènes de Polin, Mayol et Dranem. 
Etablissements Gaumont

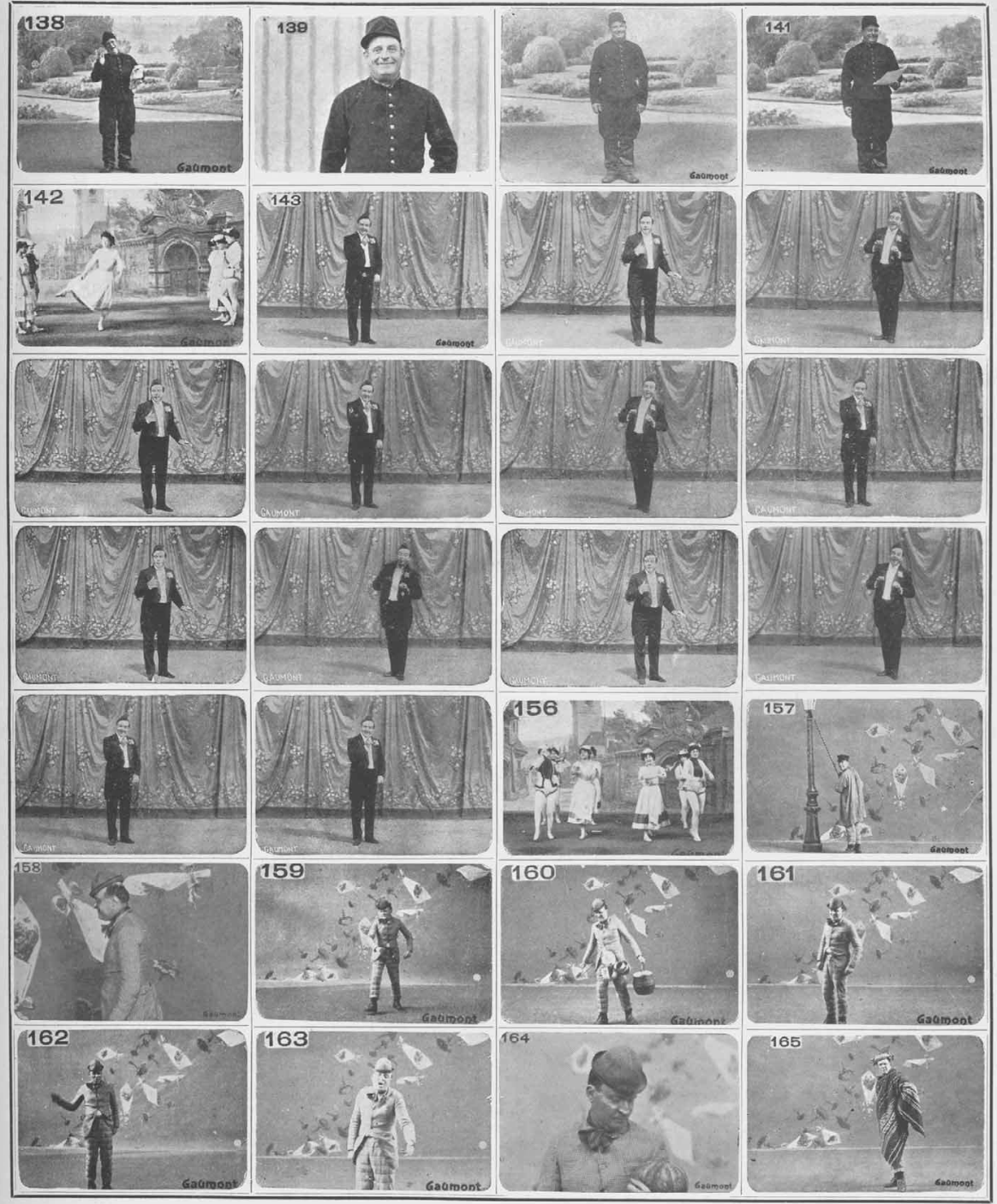

1895 /

$\mathrm{n}^{\circ} 61$

septembre

2010

\section{1}

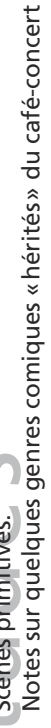


est le résultat ? Jamais ils n'ont gagné autant d'argent ; nous en connaissons, et non des moindres, qui triplent annuellement le revenu qu'ils tirent seulement de leur apparition sur les scènes ou de leurs auditions en public. ${ }^{14}$

Reste à identifier ces artistes interprètes, rarement compositeurs ou auteurs, que Gaumont tient à rassurer et ceux qui auraient triplé leurs revenus. Parmi eux, sans doute, les trois célébrités comiques de la nouvelle génération: Mayol, "1er chanteur comique de Paris »15, Dranem, comique absurdiste et Polin, comique troupier qui, en 1906, ont participé à la fondation de I'APGA, Association Phonique des Grands Artistes. Contrairement à la génération précédente, celle des premières vedettes issues du café-concert (Thérésa, Paulus et Ouvrard père qui n'ont pas enregistré de phonogrammes), cette « seconde vague " commence sa carrière sèdent un « créateur » ou une « créatrice " permettant de situer approximativement la date de leurs émergences respectives. Une première catégorie rassemble un mouvement diffus, intimiste, relativement ancien, d'abord « montmartrois ", plus tard " rive gauche ", qui va pleinement s'épanouir avec l'écoute individualisée, grâce au phonographe puis à la TSF. Ce sont là les formes « réalistes », plus féminines, qui s'affirmeront, en chansons comme à l'écran, avec le développement " parlant » du cinématographe. Nos « scènes primitives » concernent d'autres genres, plus spécifiquement comiques et masculins. Typiques des établissements de boulevard, fortement liés au développement de l'affiche lithographique à partir de 186617, ces « types" vont circuler de films en refrain. Ce sont les genres gambilleur, paysan, poivrot, gommeux, troupier et scieur.

Le genre gambilleur est créé au Jardin Oriental de Toulouse par Paulus, « le chanteur populaire », en 1868. Pour interpréter son premier succès, les Pompiers de Nanterre, le chanteur

14 Catalogue de la Société des Établissements Gaumont. Sujets proposés à la vente en janvier 1908, p. 5. 15 Slogan d'une affiche d'Adrien Barrère.

16 Comiques-troupiers, diseuses, épileptiques, excentriques, gambilleurs, gommeux, interprètes-tyroliennes, ivrognes, patriotiques, paysans, phénomènes, réalistes-pierreuses, scieurs, sentimentaux. Serge Dillaz, la Chanson sous la Ille Republique (1870-1940), Paris, Tallandier, 1991, p. 246.

17 « Apparus en même temps, le café-concert et l'affiche se sont, sur la voie du succès, servis l'un de l'autre. Des centaines de placards sortirent des ateliers de Chéret, Choubrac ou Faria, ainsi que de ceux de Charles ou Émile Lévy. Signées ou anonymes, ces affiches étaient répandues dans Paris. " (Alain Weill (dir.), Catalogue de l'exposition Le Café-Concert, affiches de la bibliothèque du musée des arts décoratifs, Paris, UCAD, 1977, p. 8). 
demande à des figurants en uniformes de sapeurs de le rejoindre lors de chaque refrain. " Je me démenais comme un diable, dans cette chanson-scène, et c'est de ce jour que je pris l'habitude de corser les ritournelles d'un petit pas de danse expressive ", signale Paulus dans ses mémoires ${ }^{18}$. $D^{\prime}$ autres chansons-scènes suivront, leur présentation ayant été rendue possible par la modification de la réglementation qui prohibait jusque-là l'usage des costumes et des accessoires en dehors des théâtres :

Cette prodigieuse éclosion avait été rendue possible quand, en 1867, l'administration par l'intermédiaire de Camille Doucet, alors directeur des Théâtres, assouplissant la réglementation très stricte à laquelle devaient se plier les cafés-concerts (rien que l'habit ou la robe du soir pour les artistes) leur donna « le droit de s'offrir des costumes, de jouer des pièces et de se payer des intermèdes de danse et d'acrobatie. »19

Attribué à Francisque Sarcey, le qualificatif de " gambillard » va définir la chanson spectacle à succès des années 1880. Gesticulation mécanique évoquant une marionnette désarticulée, ce style de jeu entrera en résonance avec la représentation tressautante du cinématographe. Une série de chansons met en scène cette rencontre comique du mécanique et du vivant. Dans la Cinématomagite20, le cinéma est dépeint comme une maladie contagieuse :

Dans le temps j'étais employé dans la cinématographie

Mais j'y ai bien vite attrapé une drôle de maladie

À force de voir trépider

les vues que l'on donne en séance

J'peux pas m'empêcher de remuer

J'ai tout l'temps quelque chose qui danse

J'ai d'la ci-ci-ci-ci-ci-némagite

Ce même thème est repris, entre autres dans $J^{\prime a i} d u$ cinéma ${ }^{21}$, chanson qui semble avoir été

18 Paulus, Trente ans de Café-Concert, souvenirs recueillis par Octave Pradels, Paris, Société d'édition et de publications, 1906 (chapitre IV). Édition électronique disponible sur le site www.chanson.udenap.org 19 Alain Weill (dir.), op. cit., p. 7.

20 Paroles de Briollet et Lelièvre, musique V. Scotto, paru dans Paris Qui Chante, n²57, 22 décembre 1907.

21 Paroles de Bouchaud. Phonoscène (programmée au Gaumont Palace en juin 1917) visible sur le DVD offert avec Giusy Pisano et Valerie Pozner (dir.), le Muet a la parole, Paris, AFRHC, 2005. Transcription de I'auteur. 
écrite pour être chronographiée, c'est-à-dire filmée en playback grâce au Chronophone Gaumont :

Je me baladais la semaine dernière

lorsqu'un cinématographeur

Me dis « voulez-vous pour vous distraire

poser devant un ciné un quart d'heure ? "

Je consens. Devant l'appareil il me place

et me fait faire un tas de contorsions

Plus vous ferez de gestes et de grimaces, plus ça sera beau

J'ai fait des trucs et des machins

Et depuis ce temps-là crénom d'un chien

Y a pas à dire j'ai du cinéma - ha - ha - ha - ha

Ainsi qu'un peu de cinémato - ho - ho - ho -ho

Dans le cœur, dans le foie, dans le mou, dans la tête,

dans les bras, dans les jambes, dans le cou,

Ciné-cinéma, j'ai du cinématographe, de la cinématographie partout

Petite madame, je vous énerve à remuer comme ça ?

Ne me regardez pas madame !

Sinon ça vous prendra comme moi !

Vous aurez du cinéma, et demain, vous y reviendrez au cinéma

Vous avouerez que c'est cocasse,

Depuis que j'ai posé devant ce fourbi,

Je ne tiens plus une minute en place,

Je gigote le jour et la nuit

Sitôt que je sors dans la rue

Les badauds, les gosses, les passants,

Les trottins et les petites grues,

Disent en riant :

Regardez donc ce ouistiti

Qui nous fait la danse de Saint-Guy 


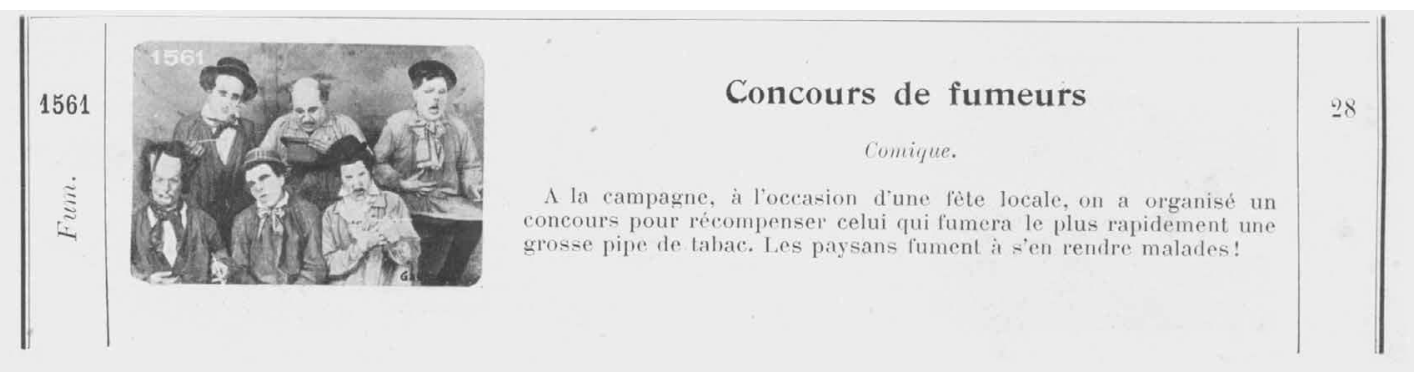

Fig. 4. Concours de fumeurs $\left(n^{\circ} 1561\right)$ Catalogue Gaumont, op. cit., 1908.

Avec la Ille République, un mode de représentation comique, "théâtralisation du quotidien 22 ", affleure. Caricaturant le réel, le café-concert projette son public sur scène. C'est le cas, par exemple avec le genre " paysan » dont la création est attribuée à Sulbac, à la Scala vers 1882. Certes, il n'a pas fallu attendre l'avant-dernière décennie du XIXe siècle pour que I'on moque des paysans en France. Mais chez Molière ou Beaumarchais, étaient-ils déjà le sujet central d'amusement, comme dans ce film Gaumont cité dans le catalogue de 1908 :

\section{Concours de fumeurs, $\mathrm{n}^{\circ} 1561$ (fig. 4)}

À la campagne, à l'occasion d'une fête locale, on a organisé un concours pour récompenser celui qui fumera le plus rapidement une grosse pipe de tabac. Les paysans fument à s'en rendre malades !23

La même remarque s'applique au genre « ivrogne ». Genre hélas peu documenté, personne ne se vantant de l'avoir créé. Le personnage du poivrot a été repris avec succès par Joseph Arnaud, Armand Bourgès, Clovis (1851-1910), Jules Réval, Mathias... Dans ses mémoires publiées en 1929, Mayol affirme avoir créé vers 1894 « un rôle de poivrot, dont [il a] conservé la photographie : on y croirait voir Charlie Chaplin, I'admirable et universel "Charlot" du cinéma actuel ! "24 La ressemblance lui paraît " assez amusante pour être signalée... ». Le genre poivrot semble avoir été en vogue chez Gaumont ainsi qu'en témoignent deux bandes toujours proposées à la vente en 1908 :

22 Concetta Condemi, les Cafés-concerts, Histoire d'un divertissement (1849-1914), Paris, Éditions Quai Voltaire Histoire, 1992, p. 176.

23 Catalogue de la Société des Établissements Gaumont, op. cit., p. 112.

24 Mayol, Mémoires, Louis Querelle Éditeur, 1929, chapitre V («L’Essor»). Édition électronique disponible sur le site http://www.chanson.udenap.org 


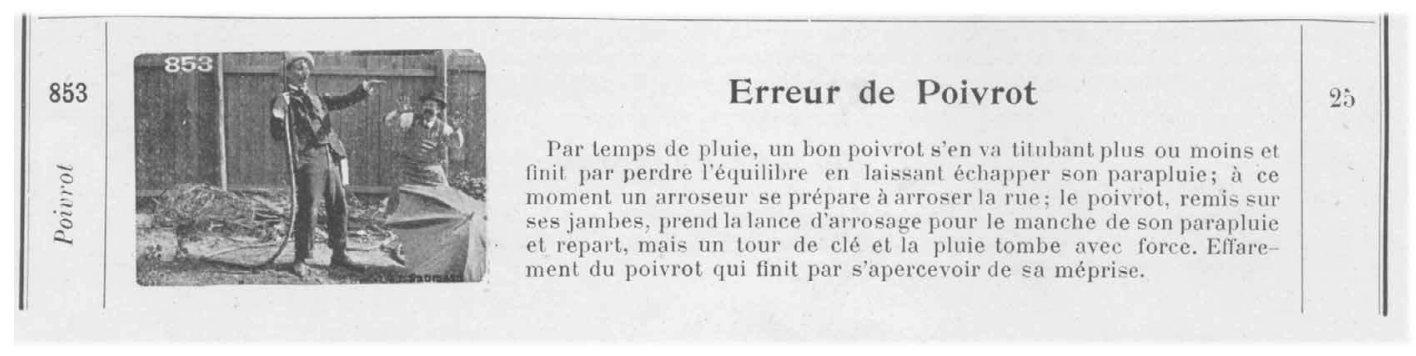

Fig. 5. Erreur de Poivrot ( $\left.n^{\circ} 853\right)$.

Catalogue Gaumont, op. cit., 1908.

\section{Erreur de Poivrot, $\mathrm{n}^{\circ} 853$ (fig. 5)}

Par temps de pluie, un bon poivrot s'en va titubant plus ou moins et finit par perdre l'équilibre en laissant échapper son parapluie ; à ce moment un arroseur se prépare à arroser la rue ; le poivrot, remis sur ses jambes, prend la lance d'arrosage pour le manche de son parapluie et repart, mais un tour de clé et la pluie tombe avec force. Effarement du poivrot qui finit par s'apercevoir de sa méprise. ${ }^{25}$

\section{On est poivrot, mais on a du cœur, $\mathrm{n}^{\circ} 1317$ (fig. 6)}

Ayant trouvé qu'il faisait une température assoiffante, un ivrogne s'était assis à la terrasse d'un café. Il voit une petite fille très chagrinée qui cherche en vain la pièce que sa mère lui a confiée pour l'achat d'un litre de vin. Dans un élan de générosité, notre pochard lui donne le litre placé sur la table, en y ajoutant le verre qu'il allait vider. ${ }^{26}$

Le genre gommeux a été créé (selon Paulus et Ouvrard) par Libert vers 1876. Personnification d'une figure populaire plus ancienne, celle du jeune homme riche, dilettante, peigné avec la raie au milieu, le genre sera notamment repris par le personnage de Max Linder. Notons que Libert quitte la scène en 1893, emportant la vogue de son genre avec lui plus de dix ans avant que Max Linder ne présente son personnage, ici décrit par Sadoul :

Le succès qui lança le personnage de Max fut les Débuts d'un patineur, tourné pendant l'hiver 1906-1907 sur le lac de Vincennes.[...]

25 Catalogue de la Société des Établissements Gaumont, op. cit., p. 82. 26 lbid., p. 93. 


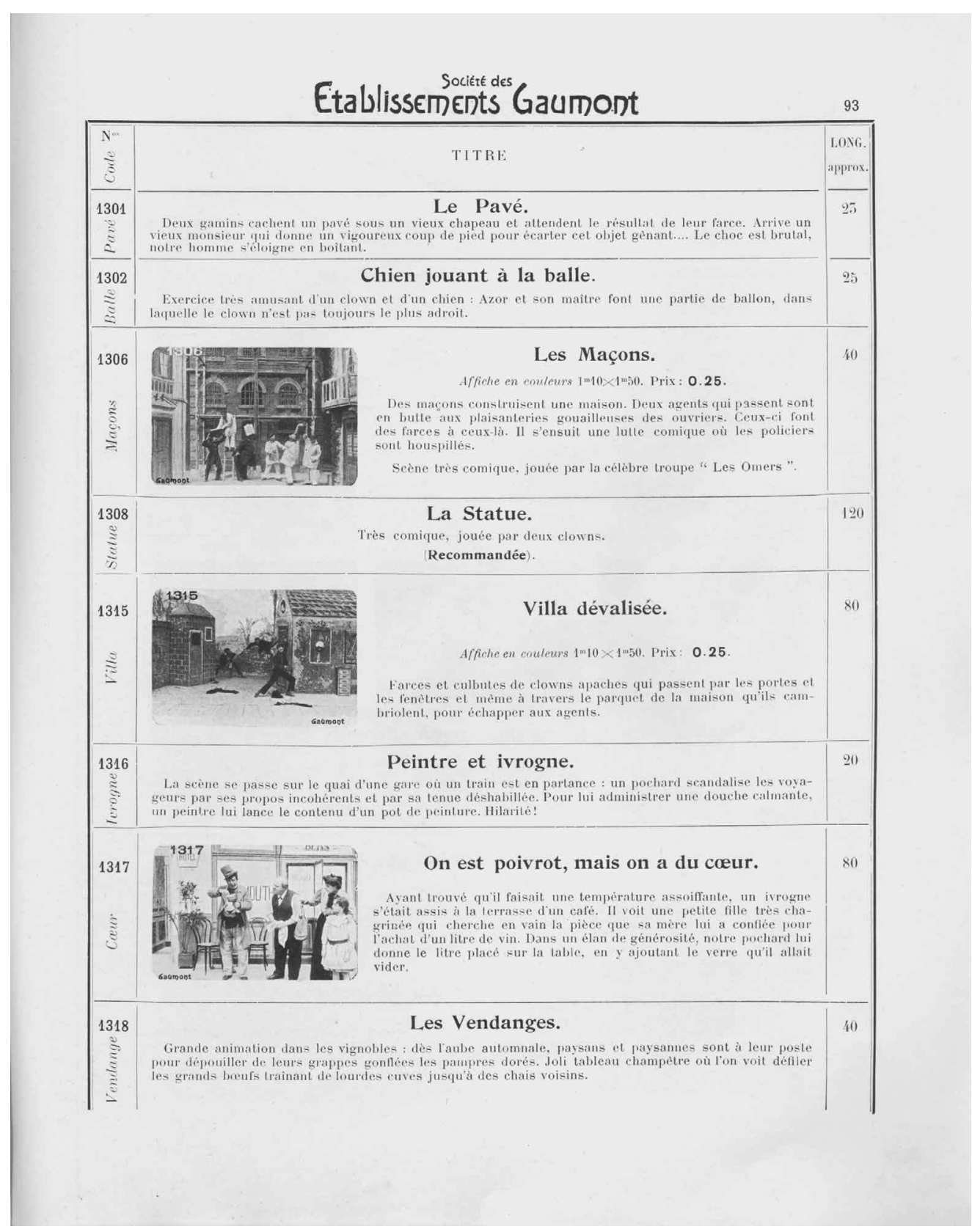

Fig. 6. On est poivrot, mais on a du cœur ( $\left.n^{\circ} 1317\right)$. Catalogue Gaumont, op. cit., 1908. 
Max Linder apparaissait sur la glace dans le costume qui devint traditionnellement le sien, et qu'il avait aussi adopté, à la même époque, dans le Pendu, d'après la chanson célèbre de Mac Nab. [...]

La distinction parfaite des gentlemen habillés, selon la publicité, à la Belle Jardinière ou au High Life Taylor, le chic d'Edmond Rostand ou d'Émile Deschanel, rendent plus comiques les chutes, les tartes à la crème, les soulographies, les coups de pied au cul. ${ }^{27}$

La création du genre " comique-troupier » (dit aussi " tourlourou ") est attribuée à Eloi Ouvrard (Bordeaux, 1855 - Bergerac, 1938). Dès 1877, il se fait connaître à Paris avec son interprétation de I'Invalide à la tête de bois (chanson d'Étienne Tréfeu et de Maximilien Gra-

Outre le propre fils d'Ouvrard, qui reprend le flambeau en 1909, un régiment de chanteurs va s'engager dans la voie du comique-troupier. Les plus connus sont Charles-Joseph Pasquier dit Bach, créateur de la Madelon, de Avec Bidasse et de la Caissière du Grand Café ainsi que Pierre-Paul Marsalès, dit Polin, créateur de la Petite Tonkinoise. Le comique de Polin va strictement à contre-courant de la vogue des années 1880. Au lieu de " gambiller » il reste planté en scène. Cette attitude statique peut être rapprochée de la description d'un film Gaumont :

\section{Le Képi, n¹154 (fig. 7)}

Le caporal apprend au soldat Cabochard le maniement d'armes. Le sergent arrive et, trouvant que Cabochard a mal mis son képi, le lui enfonce jusqu'aux oreilles et punit le caporal. Le même manège se reproduit avec l'adjudant qui place le képi de Cabochard sur les yeux, avec le sous-lieutenant qui l'incline à gauche, le capitaine qui le met en arrière, le colonel qui le redresse et enfin le général qui exige le port de la jugulaire. ${ }^{29}$

27 Georges Sadoul, Histoire générale du cinéma, tome 2, Les Pionniers du cinéma (De Méliès à Pathé), Paris, Denoël, 1947, p. 354.

28 Serge Dillaz, la Chanson sous la Troisième République (1870-1940), op. cit., p. 35.

29 Catalogue de la Société des Établissements Gaumont, op. cit., p. 89. 


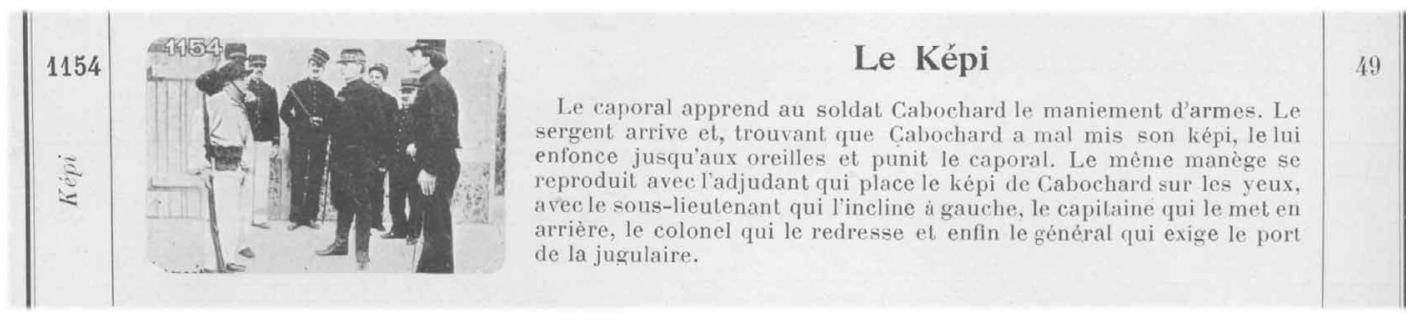

Fig. 7. Le Képi, $\mathrm{n}^{\circ} 1154$. Catalogue Gaumont, op. cit., 1908.

Combinée avec l'analphabétisme, comme c'est le cas dans l'Anatomie du conscrit (phonoscène $n^{\circ} 136$ ), et la bêtise du tourlourou (voir la Balance automatique, phonoscène $n^{\circ} 131$ ), la répétition est un des procédés les plus utilisés par le comique-troupier. Mais la pure répétition, lancinante, obstinée, a constitué en elle-même avec la " scie » un genre spécifique du café-concert. Léo Claretie en donnait en 1894 la définition suivante : « La scie populaire est un genre qui se distingue par l'incohérence voulue ou par la répétition lancinante... le résultat à atteindre est plutôt une sorte d'agacement nerveux " $^{30}$. Le recours aux onomatopées, aux expressions monosyllabiques, l'usage des allitérations caractérisent ce style. Avec les $P^{\prime}$ tits pois, Dranem pousse jusqu'à l'absurde la logique commerciale de la scie pour atteindre en quelque sorte " l'automatisme psychique » des surréalistes. En effet, dans une émission du Club d'Essai, en 1953, Raymond Queneau reconnaît que Dranem a inspiré son poème « Maigrir » (Moi, j'maigris du bout des doigts, du bout des doigts...) :

Je ne cacherai pas le goût que j'ai pour les œuvres qu'il chantait, je révélerai même que ce n'est pas dans ma famille que j'ai pris ce goût, mais bien chez Breton - j'entends : André Breton - qui régalait souvent les membres du groupe surréaliste d'un petit récital Dranem, de disques de Dranem, toujours fort apprécié. ${ }^{31}$

La trajectoire de Dranem est exceptionnelle. En 1910, il joue, dirigé par Antoine, le Médecin malgré lui à l'Odéon. Dans les années vingt, il se tourne vers l'opérette, puis vers le cinéma sonore. II meurt en 1935 au sommet de sa célébrité puisque Bardèche et Brasillach le citent dans la première phrase de leur collaboration :

Les spectateurs qui attendent d'être admis à l'intérieur de ces salles somptueuses où l'on applau-

30 Cité par Alain Weill (dir.), op. cit., p. 17.

31 Cité par François Caradec et Alain Weill, le Café-concert, op. cit., p.130. 
dit Greta Garbo et Dranem ont tous oublié, s'ils les ont jamais connus, les temps héroïques du cinéma. 32

Ironie involontaire d'une formulation équivoque : Dranem est justement un des acteurs importants - dans tous les sens du terme - de ces fameux temps héroïques. "Tantôt mitron, tantôt réserviste, quelquefois sous les traits d'une vieille femme, ailleurs en malade »33, on le retrouve à la fois dans une série de bandes dans les catalogues Pathé (le Salut de Dranem, 1901 ; Amateur de glace ; la Purge, 1904 ; le Tondeur galant ; le Séduisant barbier, 1912) et chez Gaumont pour douze phonoscènes et le Rêve de Dranem en 1905. De 1899 à 1920, il se fixe à l'Eldorado. Sur ce point, son cas n'a rien d'exceptionnel. En ce début de siècle, Polin restera pendant vingt ans à la Scala quand Mayol tiendra l'affiche du Concert parisien qui deviendra justement le Concert Mayol. Or, cette sédentarisation des artistes est pratiquement contemporaine de celle du cinéma. Nous la mettons en relation avec la mise en service des lignes du métropolitain. En quelques années, cette innovation (que Londres connaissait déjà depuis plusieurs décennies) bouleverse l'économie du spectacle parisien.

L'avènement de l'industrie cinématographique s'inscrit dans un processus plus large de rationalisation du divertissement. Cinéma, café-concert et music-hall possèdent des trajectoires parallèles que les genres comiques permettent de relier. L'histoire de ces " scènes primitives » est le lieu d'une circulation intermédiale.

32 Robert Brasillach, Euvres Complètes, tome X, Paris, Au club de l'honnête homme, 1964, p. 15 (Histoire du cinéma, reprise de l'édition de 1935).

33 Ibid., p. 31. 